\title{
Radium 223 combined with new hormone therapies for the treatment of castrate-resistant metastatic prostate cancer: scientific evidence and sharing of our experience
}

\author{
Miguel E. Jiménez-Romero ${ }^{1}$, Elba Y. Canelón-Castillo ${ }^{1}$, Sara Díez-Farto ${ }^{1}$, Jose D. Santotoribio ${ }^{2}$ \\ ${ }^{1}$ Department of Urology, ${ }^{2}$ Department of Laboratory Medicine, Puerto Real University Hospital, Cadiz, Spain \\ Correspondence to: Dr. Miguel E. Jiménez-Romero. Department of Urology, Puerto Real University Hospital, Carretera Nacional IV, Km. 665, 11510 \\ Puerto Real, Cadiz, Spain. Email: miguelefrenjimenez@gmail.com.
}

\begin{abstract}
Presentation of the interesting case of a patient suffering from castrate-resistant prostate cancer (CRPC) with bone metastasis, who received concomitant treatment with abiraterone acetate (AA) and radium-223. The patient experienced significant clinical improvement in his quality of life and pain relief after beginning the aforementioned treatment, without being affected by adverse toxicities. Currently, the correct selection of patients to receive radium-223 treatment is still a clinical challenge in the case of CRPC with metastasis. In this article, we discuss the future prospects of this treatment, reviewing current evidence about concomitant therapies with radium-223 and its present state, based upon the recent recommendations from the Pharmacovigilance Risk Assessment Committee (PRAC), and the data presented in the ERA-223 study. Based on our clinical experience, we provide practical orientation for the integration of this radiopharmaceutical in the therapeutic plan for this group of patients. We conclude, despite some of the positive results and our excellent experience, that it would be wise to wait for the results of the clinical trials that are studying the safety and benefits of the combined use of radium-223 with new hormone therapies. Bearing in mind that to date, the only published large-scale randomised trial that investigated the combination of AR-axis-targeted therapy with Ra-223 is negative, the harms of the combination outweighed any benefits in ERA-223. Nonetheless, in order to recommend whether or not this treatment should be used, it is essential to define the patient profile that could benefit from this therapeutic option.
\end{abstract}

Keywords: Radium chloride Ra-223; radiopharmaceutical; abiraterone acetate (AA); metastatic castrate-resistant prostate cancer (CRPC); bone metastasis

Submitted May 17, 2019. Accepted for publication Sep 10, 2019.

doi: $10.21037 /$ tau.2019.10.03

View this article at: http://dx.doi.org/10.21037/tau.2019.10.03

\section{Introduction}

Prostate cancer is the second most frequent cancer in males, and it represents around $7 \%$ of cancer-related deaths in the world. Androgen deprivation therapy (ADT) is the standard treatment for advanced prostate cancer patients. After initial response to this treatment, in most patients, the condition worsens to become castrate-resistant prostate cancer (CRPC) with radiological evidence of bone metastasis in many cases, which leads to the high morbidity linked to this condition. Since 2010, a number of therapeutic agents have materialised that extend the life of these patients [sipuleucel-T, cabazitaxel, abiraterone acetate (AA), enzalutamide (ENZ) and radium-223 dichloride (Ra-223)]. Their approval for use in a short period of time has not made evaluation of their combined use possible. However, there is some evidence of the safe combined use of Ra-223 with AA or with ENZ as a treatment. Nonetheless, at the beginning of December 2017, the European Medicines Agency (EMA) published a warning against this combined use. Hence, we present the only case-prior to 


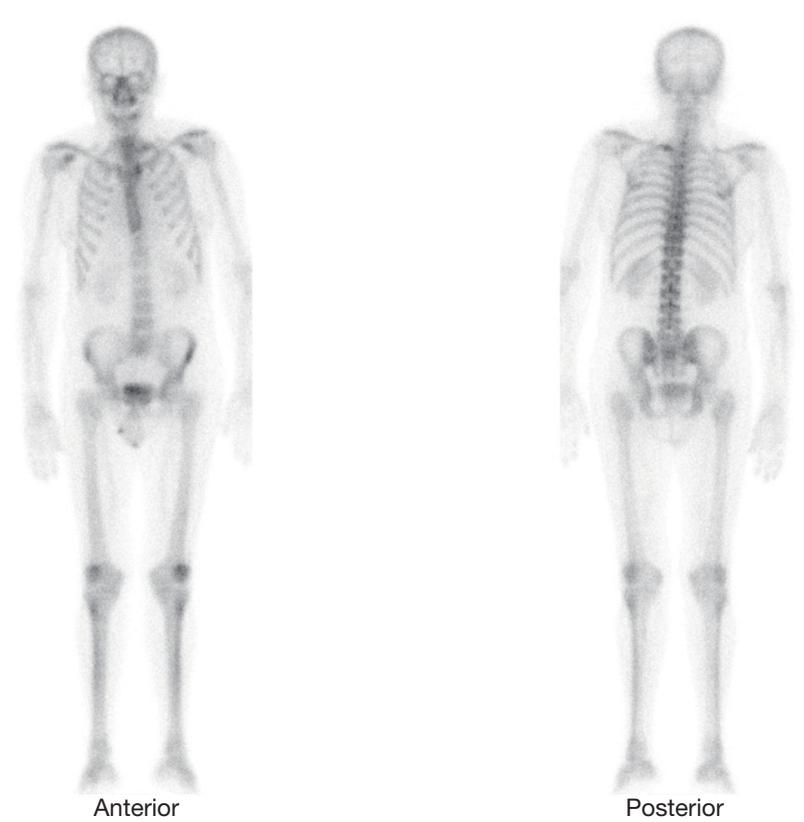

Figure 1 Bone scan from July 2013, before commencing the abiraterone acetate treatment. Showing an area compatible with metastasis located in the first left costal arch.

this warning-of the patient to whom said combination was administered. We also review the existing current bibliography related to this topic.

\section{Clinical case}

A seventy-one-year-old male patient attended our practice for the first time in August 2013, referred from a private practice. In September 2003, aged sixty-one, the patient was assessed because he presented elevated PSA levels (11.6 $\mathrm{ng} / \mathrm{mL})$. A rectal exam revealed no suspicious findings and no linked urinary symptoms. A transrectal ultrasound-guided prostate biopsy was performed with histopathological findings of prostate adenocarcinoma (ADC) Gleason $4+3$ in the left lobe, in two of the six studied cylinders. Given the prostate cancer diagnosis, clinical stage cT1c Nx Mx, intermediate risk group based on the D'Amico classification, an open radical prostatectomy was performed. The histopathological study of the surgically removed piece revealed prostate ADC Gleason $8(4+4)$ in the left lobe, affecting the prostatic capsule and both seminal vesicles (pathologic staging pT3b Nx Mx).

After this, the patient was monitored and presented undetectable PSA levels during the subsequent 17 months, until April 2005, when he presented with a PSA elevation reaching $0.22 \mathrm{ng} / \mathrm{mL}$. Suspecting that there might have been local recurrence (no radiological study was performed), a treatment of external radiation therapy linked to complete androgen blockade (CAB) was indefinitely prescribed. Said treatment was prescribed by a urologist from a private clinic who did not follow the recommendations set by the different clinical practice guidelines, nor by our action protocols, which do not recommend their indefinite use.

In subsequent follow-ups, PSA fluctuations did not exceed $2 \mathrm{ng} / \mathrm{mL}$, until June 2013 (ten years after the initial diagnosis), when, even though he had undergone $\mathrm{CAB}$ treatment, the patient presented with PSA levels of $13.25 \mathrm{ng} / \mathrm{mL}$. The patient was referred to our practice, a secondary hormonal manoeuvres treatment was begun and a computed axial tomography (CAT scan) of the abdominal pelvic region (no metastatic findings) and a bone scan were performed. The test showed an area compatible with metastasis located in the first costal arch (Figure 1).

After removal of the anti-androgen, PSA levels rose to $18.08 \mathrm{ng} / \mathrm{mL}$. The patient showed good functional state (ECOG 0) and no pain and/or asthenia. Considering the CRPC with bone metastasis (mCPRC) diagnosis, in August 2013 an AA 1,000 mg daily dose treatment was prescribed + prednisone $10 \mathrm{mg}$ daily, coupled with bone protective agents, denosumab $120 \mathrm{mg}$ monthly injection, and a calcium and vitamin D supplement.

Two weeks after the treatment was begun, the patient presented with 'PSA flare'. The drug was well tolerated without linked adverse effects. On the eighth weekly follow-up visit, we observed the first decrease in PSA levels, which was stable over time with mild fluctuations. The levels of alkaline phosphatase (ALP) and LDH were within the normal parameters, with no evidence of clinical or radiological progression found in imaging studies performed as part of the follow-up procedure. Strict quarterly follow-ups were performed with no evidence of decline during 2014 and part of 2015. In November 2015-27 months after beginning the AA treatment and 146 months after the initial cancer diagnosis-tests showed evidence of biochemical progression confirmed by very high levels of PSA $37 \mathrm{ng} / \mathrm{dL}$. These levels were not associated with clinical or radiological progression, the decision was taken to modify the corticosteroid treatment, cancelling the prednisone and beginning a daily treatment of $0.5 \mathrm{mg}$ of dexamethasone. This kept PSA levels stable at $30 \mathrm{ng} / \mathrm{dL}$, with normal analytical parameters of bone turnover and general well-being. The patient remained stable with no 


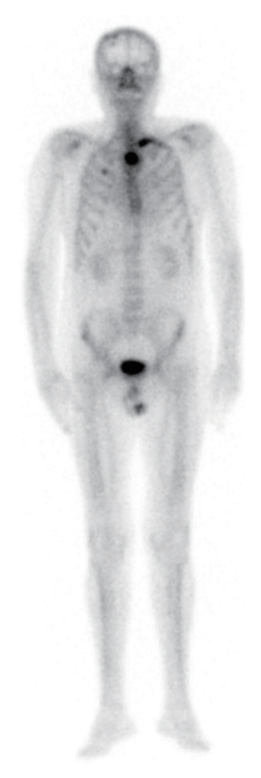

$$
\|
$$

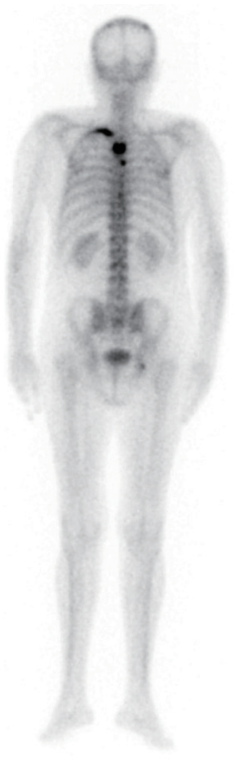

Figure 2 New bone scan from February 2017, prior to beginning the treatment with Ra-223, and 42 months after having initiated abiraterone acetate treatment. Radiological progression, with new lesions in the dorsal vertebrae and costal arch.

clinical, analytical or radiological progression during 2016, receiving quarterly follow-ups. In February 2017, after 42 months of AA treatment, an increase of the already existing lesion shown in previous bone scans was observed, as well as new lesions in the dorsal vertebrae and costal arch (Figure 2). Due to the decline in quality of life suffered by the patient, with bone pain controlled by relief medication in step two of the WHO Pain Ladder, the decision was reached to perform a positron emission tomography scan (PET-CT scan) in order to modify treatment. The scan confirmed new bone metastasis lesions, yet no measurable adenopathies or visceral lesions. Forty-five months after AA treatment initiation, and given the findings and the patient's clinical situation, the Committee for Tumours decided to discontinue treatment, as well as the calcium and vitamin D supplements. The start of Ra-223 treatment was agreed, as well as maintaining the denosumab, a bone health agent.

In May 2017, having reached a PSA level of $79.02 \mathrm{ng} / \mathrm{mL}$, an ALP level of $169 \mathrm{U} / \mathrm{L}$, and normal hematimetric values, the first dose of radiopharmaceutical was administered (a dose of $55 \mathrm{kBq} / \mathrm{kg}$ iv every four weeks providing a total of six doses). Even though the Committee reached the decision of not administering AA, the patient was presented with data showing the possible benefits of a combined treatment
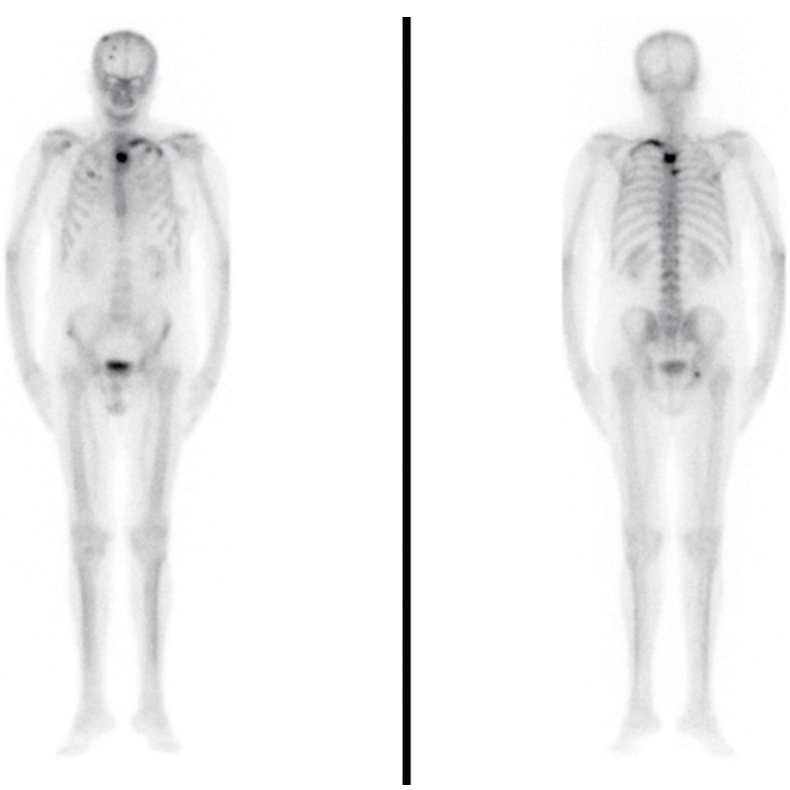

Figure 3 Bone scan from October 2017, after finishing radiopharmaceutical treatment, no radiological advance or new lesions observed.

$(\mathrm{AA}+\mathrm{Ra}-223)$. The patient consulted a foreign oncology centre for a second opinion, and, aware of the possible risks involved in the combined treatment, continued to buy and defray the costs of the AA treatment, taken in the recommended doses under our supervision. Before each Ra-223 treatment, we performed clinical and analytical tests evaluating the hematimetric values and found no evidence of cytopenias, which would contraindicate further treatment administration. After the first injection, the pain progressively decreased. When the treatment concluded in October 2017, a decrease in ALP levels to $49 \mathrm{U} / \mathrm{L}$ was observed, with PSA levels of $113.20 \mathrm{ng} / \mathrm{mL}$. The functional status of the patient remained, and an improvement of the bone pain (VAS scale) was observed, there being no need for pain medication. Once the radium-223 treatment was finalised in October 2017, we decided to continue with the AA treatment and requested a new bone scan, which concluded there was no radiological advance nor new lesions (Figure 3).

Forthwith, quarterly follow-ups were established with clinical, radiological and analysis controls, where no residual toxicity was present. In February 2018, after 54 months of castration resistance, a new bone scan showed new areas of hyperfixation in the bone, which suggested radiological progression without associated symptoms. At that point, 
an increase of PSA levels to $158 \mathrm{ng} / \mathrm{mL}$ was observed. From then to date (March 2019) the patient maintained an excellent functional status (ECOG scale 0 ), and no asthenia or bone pain. The patient's PSA rose to $456 \mathrm{ng} / \mathrm{mL}$, with $\mathrm{LDH}$ and ALP within the normal levels. In the imaging (PET-CT) scans, no new bone lesions or progression were observed.

Sixty-seven months have passed since the start of the AA treatment (nearly five and a half years), and after a new multidisciplinary assessment, the decision to maintain the current treatment was reached. The patient was given an explanation of the options for the planned treatment sequence and maintaining the current one was recommended. Follow-up visits were scheduled every two months, and the medical check-ups intensified.

\section{Discussion}

In 2013, the Food and Drug Administration (FDA) approved a radiopharmaceutical for the treatment of mCRPC with no visceral damage, which had a completely different mechanism of action to the therapies available up to then, consisting of Ra-223 dichloride (1), a radionuclide alpha-particle emitter with high lethal capacity and low tissue penetration. Given its physical properties, cancerous cells forming bone metastasis receive a high dose of radiation, resulting in an irreversible cytotoxic effect. This effect, different from the androgenic receptor pathway blockade, has proven to significantly improve the overall survival (OS) rate when administered to patients with mCRPC. It has also shown a reduction in skeletal system problems (SSPs) and in pain, all with favourable safety profiles (2). Considering all of these factors, Ra-223 has become a therapeutic option for the initial treatment of $\mathrm{mCRPC}$, as well as for refractory cases in other approved treatments. Its complementary action mechanism means it has a low probability of cross-resistance to other agents, and suggests a potential clinical advantage when combined with AA or ENZ. As well as previously mentioned, the constant search for the best available therapy leads to conducting different clinical trials with the objective of determining whether combination offers advantages regarding survival with a good tolerability profile.

Data suggests that combination does offer advantages with regards to OS rates, with favourable safety data on the use of Ra-223 with AA or ENZ. Saad et al. analysed safety and $O S$ rate in patients treated with $\mathrm{Ra}-223$ combined with other molecules (AA, ENZ or denosumab) in an international expanded access program (3). Of the 696 patients included in the study, 188 (27\%) simultaneously received Ra-223 treatment combined with other agents: 154 (22\%) with AA and 50 (7\%) with ENZ. Minor differences were present in the baseline characteristics of both groups. For instance, patients who received a concomitant treatment had lower baseline levels of PSA and ALP, as well as a better functional status. In turn, there was a higher percentage of patients who had previously been treated with docetaxel, and the time lapsed from bone metastasis diagnosis was also higher.

The average duration of combined treatment was 24.9 weeks (IQR 12.6-37.9 weeks). In the post-hoc analysis, the OS rate of the patients who had received a treatment combining Ra-223 with AA and/or ENZ (mean NA, 95\% CI, 16 months-NA) was higher than in the patients who only received a Ra-223 treatment (mean 95\% CI, 13 months, 12-16). Given that this was a non-randomised single-arm study, the differences seen in OS could be explained entirely by differences in baseline characteristics between the two groups in question. In turn, the average time until the first SSPs was 18 months. The ratio of adverse events related to the treatment was similar in both groups.

In the 2017 European Conference, the updated data of the international expanded access program was published (4), which analysed the efficiency and safety of the sequential or concomitant treatment with Ra-223 and AA. From a total of 708 patients who received $>1$ injection of $\mathrm{Ra}-223,228$ (32\%) received $\mathrm{Ra}-223$ after the AA (AA/ $\mathrm{Ra}-223)$, and 119 (17\%) received it concomitantly (Ra-223 + AA). This combination was linked to a higher tolerability profile, and presented a lower ratio of adverse effects in all levels. The median OS rate was twelve months (95\% CI, 9.7-14.3) in the AA/Ra-223 group, versus the NA (11.7$\mathrm{NA})$ in the Ra-223 + AA group.

Another recent multi-centre, open-label, international phase II trial expanded access programme was carried out in the United States (NCT01516762) on 184 patients with symptomatic mCRPC with two or more bone metastases, no hepatic, pulmonary or brain metastatic lesions, presenting interesting results (5). In this study, the group of patients included were simultaneously treated with AA or ENZ and Ra-223. In this case, 14\% (25 out of 184) of patients received a concomitant treatment with $\mathrm{AA}+\mathrm{Ra}-223$, while $8 \%$ (15 out of 184 ) received $\mathrm{ENZ}+\mathrm{Ra}-223$. Both group's characteristics were similar, except for the baseline PSA levels, which were higher in the subgroup of patients who received AA treatment. In this study, the median OS 
rate was 17 months (95\% CI, 11-NA), with 50 deaths and a high percentage of censured patients (73\%), due to a short follow-up after Ra-223 treatment approval.

The purpose of the study was also to compile additional long- and short-term safety data. The Ra-223 was well tolerated by patients who received simultaneous therapy, anaemia being the grade 3-4 most frequent side effect, which occurred in approximately $16 \%$ of patients, and thrombocytopenia in $4 \%$. In the post-hoc analysis, the Ra-223 treatment was safe whether concomitantly used with AA or ENZ. The median survival rate was higher when the patients treated with $\mathrm{Ra}-223$ were in good functional status, and in those patients who had had fewer previous treatments. The clinical benefit was higher when patients received five or six cycles of radiopharmaceutical, therefore concluding that patients with a more advanced condition had less of an opportunity to benefit from the Ra-223 treatment.

Data from another study, known as the eRADicAte (6), has also been published, which evaluates the safety of the simultaneous administration of $\mathrm{Ra}-223+\mathrm{AA}+$ prednisone in patients with symptomatic mCRPC, whether they had received previous chemotherapy treatments or not. The study was a phase II prospective trial, in which 31 patients who had concluded a 6-cycle radiopharmaceutical + AA treatment were included. The assessment was carried out using validated FACT and BPI-SF questionnaires, which evaluated the impact of the combined treatment on the quality of life and pain levels. Results showed that 20 out of 31 patients $(65 \%)$ experienced an improvement in their quality of life, and that in 18 of them (58\%), pain intensity decreased.

In March 2018, the European Medicines Agency (EMA) published a warning (7) against the concomitant use of Ra-223 and AA, which was the outcome of evaluating the results presented in a clinical trial requested by the FDA. The intention of this study was to evaluate the safety of this drug after its approval. The results of this randomised double-blind clinical trial (placebo-controlled phase 3), known as ERA-223, were presented in the ESMO 2018 by Smith et al. and later published in February 2019 $(8,9)$. Eligible patients were aged 18 years or older, and had histologically confirmed progressive, chemotherapynaïve, asymptomatic or mildly symptomatic castrationresistant prostate cancer and bone metastases. The Eastern Cooperative Oncology Group performance status being 0 or 1 , life expectancy of at least six months, and adequate haematological, renal, and liver function. They were randomly assigned (1:1) and were administered with up to six intravenous injections of Ra-223 $(55 \mathrm{kBq} / \mathrm{kg})$ or equivalent placebo every four weeks. In addition, all patients had oral administrations of $1,000 \mathrm{mg}$ of AA once a day and oral prednisone; or $5 \mathrm{mg}$ of prednisolone twice a day before and after the Ra-223 treatment or placebo. The primary purpose was symptomatic skeletal event-free survival, which was assessed with the intention to treat population. Safety analyses were performed on all patients who had been administered at least one dose of the studied drug.

Out of the 806 patients included in the clinical trial, 401 received $\mathrm{AA}+\mathrm{Ra}-223$ treatment, and the remaining $405 \mathrm{AA}+$ placebo. The trial was prematurely unblinded due to the increase in bone fractures and death in the combined treatment arm (in an unplanned $a d$-hoc analysis), the followup average not being reached. It is important to highlight that all patients finished the treatment with $\mathrm{Ra}-223$ and the follow-up continued from that point on.

Primary analysis showed that 196 (49\%) of the patients in the Ra-223 group had had at least one symptomatic skeletal problem or had died, compared to the 190 (47\%) out of 405 patients in the placebo group. Median symptomatic skeletal event-free survival was 22.3 months (95\% CI, 20.4-24.8) in the Ra-223 group and 26.0 months (21.8-28.3) in the placebo group [hazard ratio 1.122 (95\% CI, 0.9171.374); $\mathrm{P}=0.2636$.

The patients treated with $\mathrm{Ra}-223$ in combination with AA showed an increase in fracture risk (28.6\% vs. 11.4\%). While the median survival trended toward favouring the AA alone arm (30.7 vs. 33.3 months), there was not a significant difference in OS between the two arms based on the primary time-to-event analysis: hazard ratio for OS for AA + Ra-223 vs. AA + placebo was 1.195 (95\% CI, $0.950-1.505) ; \mathrm{P}=0.1280$.

The bone fracture instances were reviewed by an independent central committee. In patients pertaining to the combined group who had suffered at least one fracture confirmed by this committee (76 vs. 23, the 23 in the control group), almost half of them were linked to osteoporosis [37 (49\%) vs. 4 (4\%) for the AA group, a substantial difference]. The pathological fractures linked to bone metastasis were similar in both groups (25\% in the combined group and $26 \%$ in the control). Yet, the traumatic fractures were higher in number in the control arm of the trial (57\% vs. $36 \%$ in the combined arm). This data shed positive light on the combined AA and Ra-223 treatment with regards to its impact on the reduction of pathological and traumatic fractures; however, it was ruled out after 
observing the sharp increase in osteoporosis fracture risk (that is, fragility) in the combined arm.

The increase of fracture risk was observed especially in patients with prior osteoporosis diagnosis, and in cases presenting with fewer than six bone metastases. Additionally, it has not been possible to prove the significant benefit of the Ra-223 treatment in the patient subgroups which presented fewer than six bone metastases, or in those presenting total levels of ALP below $220 \mathrm{U} / \mathrm{L}$. This risk was reduced, but not abolished, when bone health agents (bisphosphonates or denosumab) were used. At this stage, it is also important to highlight as O'Sullivan et al. point out in a recently published Research Letter (10), that the outline imaging in these patients was not performed in ALSYMPCA and therefore it is not possible to know whether Ra-223 also caused excessive asymptomatic fractures in that trial. Hence, even if there had been a higher risk of undetected asymptomatic fractures, there is evidence of the lack of effect of radium-223 on clinically relevant fractures in the first- or second-line CRPC setting in the absence of exposure to AA. Lastly, we would like to point out that both groups presented a similar minor (grades 1-2) and major (grades 3-5) adverse events profile.

The authors firmly conclude that the addition of Ra-223 to AA plus prednisone or prednisolone did not improve symptomatic skeletal event-free survival in patients with castration-resistant prostate cancer and bone metastases, and that it was associated with an increased frequency of bone fractures compared with placebo. Thus, we do not recommend the use of this combination.

Due to the publication of these results, the EMA, in association with the PRAC, recommended that the use of Ra-223 be restricted after receiving at least two lines of treatment, or for patients for whom there was no therapeutic alternative. Similarly, the EMA recommended that this treatment not be used on asymptomatic patients with a reduced number of bone metastases (7). However, the results shown in the pivotal study ALSYMPCA (1) were not taken into consideration when making this decision, it was solely based upon the results of the ERA-223, a trial which was carried out on asymptomatic or slightly symptomatic patients who had not received other lines of treatment. This patient profile was very different from the one included in this pivotal study, which had led to the approval for use of the radiopharmaceutical.

After having thoroughly analysed the existing data, it may be possible that the combination of these drugs, as the first line of treatment in asymptomatic or slightly symptomatic patients, entails a higher toxicity risk without showing significant differences with regard to OS. In patients who received lines of treatment in a more advanced stage of the illness, the clinical advantages of using these combined molecules is yet to be determined. It is important to highlight that the increase of bone fractures could have been caused by the cumulus of bone demineralisation resulting from the use of these drugs individually (corticosteroids + $\mathrm{AA}+\mathrm{Ra}-223)$, and not from the tumour itself.

In conclusion, we would like to stress that our case does not particularly illustrate the possibility of any synergy between AA and Ra-223. But rather, a clinical and ALP response to Ra-223 given after progression on AA. AA appears to be a 'bystander' to the response to Ra-223. We also believe new clinical trials need to be carried out that reflect the reality of our patients. We do not agree with the radiopharmaceutical restriction for a third line of treatment, given that the pivotal study proved there were clear advantages for the OS rate, when using it in patients with the right profile. All these decisions considerably limit the number of patients that could benefit from Ra-223 treatment at the right stage of the illness. We strongly believe that the SSPs prevention therapies, and all the treatments linked to preserving bone metastasis patients bone health, are essential for management of the disease.

Regarding the combined use of Ra-223 with the new hormonal molecules used to treat mCRPC, studies must be carried out to understand if the sum of toxicity is more beneficial than the existing risk of accumulated toxicity after various treatment lines. Thus, conclusive and decisive data is needed to be able to recommend the combined use. Nonetheless, we believe it is prudent to wait for the results of the clinical trials already under way, which are studying the safety and benefits of this type of therapy. We are analysing additional phase II and III trials that are incorporating Ra-223 treatment, which are designed to evaluate combination with enzalutamide, abiraterone and other approved agents [ClinicalTrials.gov: NCT03574571, NCT03737370, NCT02199197, NCT03344211, NCT02194842, NCT03305224, NCT02034552 (the complete results of which were recently published) NCT03414437, NCT02034552, NCT02043678, NCT02903160].

\section{Acknowledgments}

None. 


\section{Footnote}

Conflicts of Interest: The authors have no conflicts of interest to declare.

Ethical Statement: The authors are accountable for all aspects of the work in ensuring that questions related to the accuracy or integrity of any part of the work are appropriately investigated and resolved. Written informed consent was obtained from the patient for publication of this Case Report and any accompanying images.

\section{References}

1. Parker CC, Pascoe S, Chodacki A, et al. A randomized, double-blind, dose-finding, multicenter, phase 2 study of radium chloride ( $\mathrm{Ra} 223)$ in patients with bone metastases and castration-resistant prostate cancer. Eur Urol 2013;63:189-97.

2. Parker C, Heidenreich A, Nilsson S, et al. Current approaches to incorporation of radium-223 in clinical practice. Prostate Cancer Prostatic Dis 2018;21:37-47.

3. Saad F, Carles J, Gillessen S, et al. Radium-223 and concomitant therapies in patients with metastatic castration-resistant prostate cancer: an international, early access, open-label, single-arm phase $3 \mathrm{~b}$ trial. Lancet Oncol 2016;17:1306-16.

4. Saad F, Heinrich D, Gillessen S, et al. Radium-223 (Ra223) in sequence or in concurrent use with abiraterone acetate (AA) or enzalutamide (E) in metastatic castration resistant prostate cancer (mCRPC) patients treated in an international early access program (iEAP). [Abstract] En:

Cite this article as: Jiménez-Romero ME, Canelón-Castillo EY, Díez-Farto S, Santotoribio JD. Radium 223 combined with new hormone therapies for the treatment of castrate-resistant metastatic prostate cancer: scientific evidence and sharing of our experience. Transl Androl Urol 2019;8(5):567-573. doi:10.21037/tau.2019.10.03 32nd Annual EAU Congress. London, United Kingdom: Elsevier Science; 2017:e94.

5. Sartor O, Vogelzang NJ, Sweeney C, et al. Radium-223 Safety, Efficacy, and Concurrent Use with Abiraterone or Enzalutamide: First U.S. Experience from an Expanded Access Program. Oncologist 2018;23:193-202.

6. Shore ND, Tutrone RF, Mariados NF, et al. eRADicAte: A Prospective Evaluation Combining Radium-223 Dichloride and Abiraterone Acetate Plus Prednisone in Patients With Castration-Resistant Prostate Cancer. Clin Genitourin Cancer 2018;16:149-54.

7. European Medicines Agency. EMA restricts use of prostate cancer medicine Xofigo. Available online: www.ema. europa.eu/medicines/human/referrals/xofigo

8. Smith MR, Parker CC, Saad F, et al. 2018, ERA 223: A phase 3 trial of radium-223 (Ra-223) in combination with abiraterone acetate and prednisone/prednisolone for the treatment of asymptomatic or mildly symptomatic chemotherapynaïve patients with mCRPC. European Society for Medical Oncology Congress, Munich, 19-23 October.

9. Smith M, Parker C, Saad F, et al. Addition of radium-223 to abiraterone acetate and prednisone or prednisolone in patients with castration-resistant prostate cancer and bone metastases (ERA 223): a randomised, doubleblind, placebo-controlled, phase 3 trial. Lancet Oncol 2019;20:408-19.

10. O'Sullivan JM, Heinrich D, James ND, et al. The Case Against the European Medicines Agency's Change to the Label for Radium-223 for the Treatment of Metastatic Castration-resistant Prostate Cancer. Eur Urol 2019;75:e51-2. 